\title{
Perfil das doadoras de leite materno de um banco de leite humano de um hospital universitário do sul do Brasil
}

\author{
Profile of breast milk donors from a human milk bank from a south Brazil university hospital \\ Perfil de donantes de leche materna de un banco de leche humana en un hospital universitario en el
} sur de Brasil

Recebido: 28/12/2021 | Revisado: 03/01/2022 | Aceito: 08/01/2022 | Publicado: 12/01/2022

\author{
Rosana Loureiro \\ ORCID: https://orcid.org/0000-0002-8734-3510 \\ Universidade Católica de Pelotas, Brasil \\ E-mail: rosana.loureiro@hotmail.com \\ Vera Lúcia Marques de Figueiredo \\ ORCID: https://orcid.org/0000-0002-3580-0804 \\ Universidade Católica de Pelotas, Brasil \\ E-mail: verafig@terra.com.br \\ Cristian Dornelles \\ ORCID: https://orcid.org/0000-0001-9002-613X \\ Universidade Católica de Pelotas, Brasil \\ E-mail: dornelles.cristian@gmail.com \\ Joycianne Ramos Vasconcelos de Aguiar \\ ORCID: https://orcid.org/0000-0002-0203-4939 \\ Universidade Católica de Pelotas, Brasil \\ E-mail: joycianneaguiar@hotmail.com
}

\begin{abstract}
Resumo
Objetivo: identificar o perfil segundo as variáveis sociodemográficas e perinatais das doadoras de leite materno de um banco de leite humano no Sul do Brasil. Métodos: estudo transversal, descritivo, realizado com base em 200 fichas cadastrais de mulheres doadoras no ano de 2018. Os dados coletados foram tabulados e posteriormente analisados por meio de estatísticas descritivas. Resultados: Identificou-se que as doadoras tinham mais de 25 anos (57,5\%), ensino médio $(42,5 \%)$ e exerciam alguma atividade profissional (50,5\%). A maioria realizou suas consultas de pré-natal em instituições públicas $(64,5 \%)$, eram multíparas $(58,5 \%)$, com bebês nascidos atermo (58\%), de cesariana $(59,5 \%)$ com peso adequado ao nascer $(64,5 \%)$. Não possuíam experiência em gestação anterior com amamentação (56,5\%) e receberam orientação sobre amamentação no pré-natal $(64,5 \%)$. No que se refere, as informações sobre o BLH, a maior parte das doadoras, recebeu essas informações de profissionais de saúde do hospital universitário (49,5\%). Conclusão: Tendo em vista os benefícios trazidos pelo BLH, é de extrema importância conhecer as características das doadoras de leite humano, as quais poderão nortear os gestores na implementação de estratégias de intervenção direcionadas as ações educativas ede promoção ao aleitamento materno. Essas políticas poderão aumentar o número de doadoras e o volume de leite coletado.
\end{abstract}

Palavras-chave: Bancos de leite; Doadoras de leite humano; Aleitamento materno; Amamentação.

\begin{abstract}
Objective: to identify the profile according to the sociodemographic and perinatal variables ofbreast milk donors from an human bank milk in southern Brazil. Methods: cross-sectional, descriptive study, carried out based on 200 records of women donors in 2018. The data collected were tabulated and subsequently analyzed using descriptive statistics. Results: It was identified that the donors were over 25 years old $(57.5 \%)$, high school $(42.5 \%)$ and had some professional activity $(50.5 \%)$. Most held their prenatal consultations in public institutions $(64.5 \%)$, were multiparous $(58.5 \%)$, with babies born at term (58\%), cesarean section $(59.5 \%)$ with weight appropriate to the be born $(64.5 \%)$. They had no experience of previous pregnancy with breastfeeding $(56.5 \%)$ and received guidance on breastfeeding in prenatal care $(64.5 \%)$. With regard to information about the HMB, most donors received this information from college hospital health professionals $(49,5 \%)$. Conclusion: In view of the benefits brought by the HMB, it is extremely important to know the characteristics of human milk donors, which may guide managers in the implementation of intervention strategies aimed at educational actions and promotion of breastfeeding. These policies may increase the number of donors and the volume of milk collected.
\end{abstract}

Keywords: Milk banks; Human milk donors; Breast feeding; Breast feeding. 


\begin{abstract}
Resumen
Objetivo: identificar el perfil según variables sociodemográficas y perinatales de donantes de leche materna de un banco de leche humana del sur de Brasil. Métodos: estudio descriptivo transversal, basado en 200 formularios de registro de mujeres donantes en el año 2018. Los datos recolectados fueron tabulados y posteriormente analizados mediante estadística descriptiva. Resultados: Se identificó que los donantes eran mayores de 25 años (57,5\%), tenían estudios secundarios (42,5\%) y tenían alguna actividad profesional (50,5\%). La mayoría acudió a sus consultas prenatales en instituciones públicas $(64,5 \%)$, fueron multíparas $(58,5 \%)$, con recién nacidos a término $(58 \%)$, por cesárea $(59,5 \%)$ con peso adecuado para los nacidos $(64,5 \%)$. No tenían experiencia en un embarazo anterior con lactancia materna $(56,5 \%)$ y recibieron orientación sobre lactancia materna en el período prenatal $(64,5 \%)$. En cuanto a la información sobre el $\mathrm{HMB}$, la mayoría de los donantes recibieron esta información de los profesionales sanitarios del hospital universitario (49,5\%). Conclusiones: Ante los beneficios que aporta el HMB, es de suma importancia conocer las características de los donantes de leche humana, que pueden orientar a los gestores en la implementación de estrategias de intervención orientadas a acciones educativas y promoción del amamantamiento. Estas políticas pueden aumentar el número de donantes y el volumen de leche recolectada.
\end{abstract}

Palabras clave: Bancos de leche; Donantes de leche materna; Lactancia materna; Lactancia materna.

\title{
1. Introdução
}

A ciência, com a sua unanimidade, reconhece o leite materno como fonte segura de nutrição, para o humano, no início de sua vida, cujos benefícios refletem na idade adulta (Daniels et.al., 2017; Dawod \& Marshall, 2019). Ainda, conforme os mesmos autores, os benefícios do leite materno já são bem documentadose são de suma importância para o desenvolvimento saudável das populações. Os índices de mortalidade com a amamentação são reduzidos em até $60 \%$ para os casos de infecção respiratória e $80 \%$ para os casos de diarreia, as duas principais causas de óbito no período neonatal precoce (Nunes, 2015). A recomendação da amamentação materna, para prematuros tem sido defendida com base em suas propriedades imunológicas, contribuição para maturação gastrointestinal e formação do vínculo entre mãe e filho (Brasil, 2015). A literatura salienta, também, que a amamentação é um dos fatores mais importantes para o desenvolvimento emocional dos recém-nascidos (RN's), de modo a facilitar mais tarde, o seu relacionamento interpessoal e ainda contribuir para o seu desenvolvimento psicomotor (Pereira et. al., 2020; Salvatori et. al., 2021).

A Organização Mundial de Saúde (OMS) e o Ministério da Saúde (MS) do Brasil recomendam a amamentação materna (AM) por dois anos ou mais e exclusivo nos primeiros seis meses de vida (Brasil, 2015). Em 1986, o aleitamento materno exclusivo em RN's, menores de 6 meses, era praticamente inexistente (5\%) e atingiu 37\% em 2013, devido às intervenções nas comunidades, aos serviçosde saúde, ao contexto político e econômico no Brasil. As ações da Política Nacional de Aleitamento Materno levaram o Brasil, em 2016, a ser reconhecido em seus avanços na taxa dealeitamento materno, tornando-se referência numa comparação com cerca de 150 países (Leal et.al., 2018).

Os Bancos de Leite Humano (BLH) são parte de uma importante estratégia governamental voltada para saúde infantil, com foco especial nos recém-nascidos prematuros internados em unidades hospitalares. As raízes dos BLH remetem a 2250 anos antes de Cristo,momento em que se praticava o wet nurse, em que as crianças eram amamentadas pelas suas mães, amigas, familiares, ou estranhas, que tivessem os atributos necessários de boas doadoras (Fang et. al., 2021).

A criação dos bancos de leite data de 1900, na Áustria, se estendendo para o Reino Unido e Estados Unidos (Maia et. al., 2006). No Brasil, iniciou-se em 1947, no Rio de Janeiro, com o objetivo de recolher e distribuir leite humano, visando atender os casos especiais, como prematuridade, distúrbios nutricionais e alergias a proteínas (Maia et. al., 2006). Os bancos são responsáveis por ações de incentivo ao AM,coleta da produção láctea da nutriz, seleção, classificação, processamento, controle de qualidade e distribuição (Anvisa, 2016).

Na década de 1980 foram criadas e publicadas as primeiras normas técnicas com o objetivo de monitorar a implementação e o funcionamento dos BLH, sustentados por trabalho de pesquisa e de desenvolvimento tecnológico de forma a otimizar as condições operacionais dos BLH em todo o mundo (Pereira et. al., 2020). 
Neste cenário, a participação da doadora de leite humano é extremamente importante, já que os BLH são instituições públicas que não visam lucros e somente com o auxílio das doadoras, estes podem cumprir o seu papel de forma a suprir as necessidades de seus receptores (Maia et. al., 2006; Lourenço, 2012). Segundo o Ministério da Saúde, as mulheres devem se dispor a doar, por livre e espontânea vontade, o excesso de leite produzido (Anvisa, 2016).

Para serem doadoras, as mulheres devem atender os seguintes critérios: estar amamentando ou ordenhando leite humano para o próprio filho, para doar exclusivamente o excedente; ser saudável; apresentar exames pré ou pós-natal compatíveis com a doação de leite humano; não fumar mais de 10 cigarros por dia; não usar medicamentos incompatíveis com a amamentação; não usar álcool ou drogas ilícitas; realizar exames quando o cartão de pré-natal não estiver disponível ou a nutriz não tiver realizado pré-natal e realizar outros exames conforme o perfil epidemiológico local ou individual da doadora (Neia et. al., 2021).

A seleção das doadoras é uma atribuição do médico responsável pelas atividades médico-assistenciais do BLH tendo atendido aos requisitos, será preenchido o formulário de cadastro. Nele constam dados de identificação da doadora e do RN, como: idade, endereço, informações sobre o pré-natal, parto e intercorrências neste período, peso e altura, além de resultados de exames laboratoriais, especialmente os sorológicos. Constam ainda, informações socioeconômicas sobre as doadoras, importantes para assegurar que as mesmas dispõem de condições mínimas para extração e conservação do leite a ser doado, como: presença de água encanada no domicílio, freezer e/ou congelador para o armazenamento adequado e manutenção da temperatura de congelamento do leite, garantindo as propriedades do leite humano até chegar ao receptor final (Anvisa, 2016).

Sabe-se que cada população tem a sua particularidade no processo de amamentação e doação de leite humano, bem como no perfil da doadora, por isso a importância de conhecer cada realidade, para que medidas específicas sejam tomadas. Baseado na literatura, as doadoras de outras regiões do sul do país, sudeste e nordeste, caracterizam-se por ser na sua maioria primíparas, trabalhadoras, com idade entre 20-34 anos, com ensino superior; realizaram o pré-natal completo na rede privada e tiveram seus bebês a termo, com peso adequado, de parto cesárea No entanto, no que se refere a informações sobre amamentação e BLH, as fontes de informação são variadas, vão desde familiares, amigos e profissionais de saúde até informações recebidas pela mídia (Maia et. al., 2006; Lourenço, 2012; Machado, 2017).

É de extrema importância conhecer as características das doadoras de leite humano, as quais poderão nortear os gestores na implementação de estratégias de intervenção direcionadas as ações educativas e de promoção ao aleitamento materno. Essas políticas poderão aumentar o número de doadoras e o volume de leite coletado. O objetivo do presente estudo, foi identificar o perfil sociodemográfico e perinatal das doadoras de leite materno de um BLH no sul do Brasil, comoforma de promoção e apoio ao aleitamento materno e possível captação de doadoras.

\section{Metodologia}

Trata-se de um estudo descritivo, com abordagem quantitativa, realizado no Banco de Leite Humano (BLH) de um hospital universitário no sul do Brasil, que ocorreu no período de janeiro/2018 a dezembro/2018.

O BLH presta atendimento às parturientes da maternidade e às nutrizes externas e é responsável pela promoção, proteção e apoio ao aleitamento materno, além da execução das atividades de coleta, processamento e controle de qualidade do leite humano, para posteriormente distribuir aos recém-nascidos que necessitarem, sob prescrição médica, atendendo toda a demanda do hospital universitário da região.

O estudo é composto por uma amostra de 200 nutrizes. Foram utilizados cadastros das doadoras externas ao BLH, caracterizando aquelas nutrizes que fazem ordenha domiciliar do leite, as mesmas recebem visitas em casa do funcionário do BLH e a outra parte da amostragem é caracterizada por doadoras internas, aquelas que são atendidas no próprio BLH do hospital universitário. Foi excluído do estudo, quatro fichas cadastrais incompletas e/ou com letra ilegível. 
Os dados foram coletados de forma retrospectiva, pela própria pesquisadora, através de dados secundários das fichas cadastrais das doadoras aptas do BLH do hospital, preenchidas pelas funcionárias do setor, revisadas pela nutricionista, responsável técnica e pelo médico responsável pelo BLH, para liberação da coleta do leite materno.

Para cada nutriz foi preenchida uma ficha, contendo variáveis de dados sociodemográficas (idade materna, escolaridade e profissão) e dados perinatais (número de gestações, idade gestacional do recém-nascido, peso ao nascer, tipo de parto, local do pré-natal, número de consultas de pré-natal, experiência com amamentação, orientações sobre amamentação e fonte de informação sobre o BLH).

A análise estatística descritiva foi realizada através do Statistical Package for the Social Sciences (SPSS), versão 26.0, por meio de freqüências absolutas e relativas. A pesquisa foi aprovada pelo Comitê de Ética em Pesquisa, sob o parecer $n^{\circ}$.3.975.735. Por se tratar da coleta de dados secundários, não foi necessária a aplicação do Termo de Consentimento Livre e Esclarecido e não houve a identificação dos participantes, conforme a resolução 466/2012 do Conselho Nacional de Saúde.

\section{Resultados}

O estudo possibilitou conhecer as características das doadoras de leite humano. A Tabela 1 mostra a distribuição das nutrizes segundo as variáveis sociodemográficas. A maioria das doadoras tinham mais que 25 anos (57,5\%), ensino médio completo ou incompleto $(42,5 \%)$ e exerciam uma atividade profissional $(50,5 \%)$.

Tabela 1. Distribuição das doadoras segundo características sociodemográficas, 2018, RS.

\begin{tabular}{lll}
\hline Variáveis & $\mathbf{N = 2 0 0}$ & $\mathbf{\%}$ \\
\hline Faixa Etária & & \\
$<18$ anos & 16 & 8,0 \\
18 a 25 anos & 69 & 34,5 \\
$>25$ anos & 115 & 57,5 \\
Escolaridade & & \\
Não alfabetizada & 1 & 0,5 \\
Ensino fundamental completo/incompleto & 78 & 39,0 \\
Ensino médio completo/incompleto & 85 & 42,5 \\
Ensino superior completo/incompleto & 36 & 18,0 \\
Atividade Profissional & & \\
Não & 99 & 49,5 \\
Sim & 101 & 50,5 \\
\hline
\end{tabular}

Fonte: Autores (2018).

A Tabela 2 apresenta as variáveis perinatais das doadoras de leite do BLH. Em relação a paridade, 58,5\% das mulheres eram multíparas, tiveram bebês a termo (37 a 41 semanas) (58\%) e os bebês nasceram com peso usual (64,5\%). A maior parte das doadoras $(59,5 \%)$ foram submetidas à cesariana, tinham pré-natal completo (6 consultas ou mais de pré-natal) $(75,5 \%)$ e o realizaram na rede pública $(64,5 \%)$. 
Tabela 2. Distribuição das doadoras quanto às informações perinatais, 2018, RS.

\begin{tabular}{|c|c|c|}
\hline Variáveis & $\mathrm{N}=\mathbf{2 0 0}$ & $\%$ \\
\hline \multicolumn{3}{|l|}{ Número de gestações } \\
\hline primípara & 83 & 41,5 \\
\hline multípara & 117 & 58,5 \\
\hline \multicolumn{3}{|l|}{ Idade gestacional } \\
\hline pré-termo & 84 & 42,0 \\
\hline a termo & 116 & 58,0 \\
\hline \multicolumn{3}{|l|}{ Peso ao nascer } \\
\hline extremo baixo peso $(<1000 \mathrm{~g})$ & 6 & 3,0 \\
\hline muito baixo peso (1000-1499g) & 15 & 7,5 \\
\hline baixo peso (1500-2500g) & 50 & 25,0 \\
\hline peso usual (2501-5500g) & 129 & 64,5 \\
\hline \multicolumn{3}{|l|}{ Tipo de parto } \\
\hline vaginal & 81 & 40,5 \\
\hline cesárea & 119 & 59,5 \\
\hline \multicolumn{3}{|l|}{ Local do pré-natal } \\
\hline público & 129 & 64,5 \\
\hline privado & 71 & 35,5 \\
\hline \multicolumn{3}{|l|}{ Pré-natal } \\
\hline sem pré-natal & 5 & 2,5 \\
\hline pré-natal incompleto & 44 & 22,0 \\
\hline pré-natal completo & 151 & 75,5 \\
\hline \multicolumn{3}{|l|}{ Experiência com amamentação } \\
\hline não & 113 & 56,5 \\
\hline $\operatorname{sim}$ & 87 & 43,5 \\
\hline
\end{tabular}

Fonte: Autores (2018).

A Tabela 3, exibe as variáveis quanto a orientações sobre amamentação recebidas no pré-natal, informações e a fonte de informações sobre o BLH. A maioria das doadoras receberam orientações sobre amamentação no pré-natal (64,5\%) e também receberam informações sobre o BLH (73,5\%), sendo a fonte de informação em sua maior parte, pela equipe de saúde do Hospital Universitário (enfermagem, nutricionista e assistente social) (49,5\%).

Tabela 3. Distribuição das doadoras quanto a orientações sobre amamentação recebidas ou não durante o pré-natal, informações e fonte de informações sobre o banco de leite humano. 2018, RS.

\begin{tabular}{lll}
\hline Variáveis & $\mathbf{N = 2 0 0}$ & $\mathbf{\%}$ \\
\hline Orientações sobre amamentação no pré-natal & & 35,5 \\
não & 71 & 64,5 \\
sim & 129 & 26,5 \\
Informações sobre o Banco de Leite Humano(BLH) & & 73,5 \\
não recebeu & 53 & 6,5 \\
recebeu & 147 & 49,5 \\
Fonte informação profissional sobre o BLH & & 3,0 \\
Equipe de enfermagem da UBS & 13 & 0,5 \\
Profissionais de saúde do HU & 99 & 12,0 \\
Equipe do BLH & 6 & 28,5 \\
fonoaudióloga & 1 & 24 \\
médico pediatra/obstetra & 57 & \\
Iniciativa própria & & \\
\hline
\end{tabular}




\section{Discussão}

Os bancos de leite só existem porque mulheres em lactação se prontificam em oferecer gratuitamente seu leite excedente a outros bebês após alimentarem seus filhos. Conhecer quem são as mulheres doadoras do BLH/HU, capazes de gesto tão nobre, mobilizou as autoras a realizarem este trabalho.

Do ponto de vista reprodutivo, a faixa etária de 20 a 30 anos é considerada ótima, pois apresenta menores riscos perinatais. Supõe-se que a maior prevalência de doadoras com idade acima de 25 anos, apresentada no presente estudo, pode ser justificada por ser o período de maior fertilidade e maturidade da mulher para maternidade. $\mathrm{O}$ mesmo pode ser observado em um estudo americano realizado no Texas, no qual as doadoras eram adultas jovens (66\%) (Oslaldiston, 2007). Observa-se que mulheres adultas tem maior experiência com amamentação e provavelmente preocupam-se mais com doação de leite (Machado, 2017).

Na literatura, as doadoras de leite humano, possuem instrução do ensino fundamental até o universitário, predominando o ensino superior (Nunes, 2015). Na amostra encontrada, apenas 5\% das doadoras eram analfabetas, já a maioria tinha, no mínimo, ensino médio, sendo assim, observa-se que o grau de instrução da doadora pode interferir na captação da mensagem sobre a relevância do aleitamento materno e, portanto, na decisão e adesão à prática de doação do leite humano (Machado, 2017).

Analisando a situação funcional das doadoras, um estudo, realizado na cidade de Tubarão, em Santa Catarina, concluiu que elas foram, na sua maioria, mulheres que trabalhamfora de casa (Nunes, 2015). O predomínio de mulheres doadoras que trabalham, constitui um fator positivo, neste estudo, demonstrando que, as mães trabalhadoras continuam amamentando, situação oposta à das décadas de 1940 e 1970, no primeiro Banco de Leite Humano do Instituto Fernandes Figueira, quando as doadoras eram pobres em sua totalidade, não trabalhavam, e encontravam na comercialização do leite humano uma forma de sustento (Machado, 2017).

Em relação a paridade, a presente pesquisa encontrou entre as multíparas, o maior número de doadoras, fator que vai de encontro com os resultados encontrados em outros estudos realizados no sul e no sudeste do país, onde mais da metade das doadoras eram primíparas (Nunes, 2015; Lourenço, 2012; Silva et.al., 2020). Mães multíparas, por já terem alguma experiência com amamentação, tem maior predisposiçãopara doação (Machado, 2017; Barros et. al., 2018).

Com relação ao peso de nascimento dos bebês, no presente estudo observou-se baixa frequência de doadoras com filhos nascido compeso abaixo do normal. Tal fato pode ser entendido porque os recém-nascidos com baixo pesoou peso insuficiente ao nascer tem maior necessidade de leite materno para atingir o peso adequado e com isso as mães acabam por não ter leite excedente para ser doado, somado ao fato de que muitas delas tem receio de doar o leite com medo de faltar para seu filho (Pereira et. al., 2020).

O parto normal é o recomendado para todas as mulheres pelo Ministério da Saúde,porém em função de intercorrências na gestação, ou no trabalho de parto, por vezes não podemser realizados (Brasil, 2017). O Brasil mantém há uma década uma preocupante posição de liderança mundial em partos cirúrgicos, as cesarianas possivelmente evitáveis aumentam a proporção de bebês que nascem antes da maturidade biológica, bebês quenascem com 37 ou 38 semanas de idade gestacional correm um pequeno risco de ter complicações de saúde, que, no entanto, poderiam ser evitadas com o adiamento do parto, aindasegundo os mesmos autores, o direito da mulher de optar pela cesariana, não deveria competir com o das crianças de nascer com 39 semanas ou mais (Barros et. al., 2018).

Quanto ao local das consultas e realização do pré-natal, a maioria das doadoras fizeram o pré-natal completo, na rede pública, resultado esse, semelhante a outro estudo realizado na região nordeste do Brasil (Machado, 2017). Observa-se que quando o pré-natal é realizado na rede pública as mulheres apresentaram mais chances de tornarem-se doadoras e a 
justificativa para esse fato, pode ser devido a orientações recebidas sobre aleitamento materno e o apoio que recebem dos profissionais de saúde durante o pré-natal, uma vez que os BLH estão vinculados a rede pública.

Em relação as fontes de informação sobre o BLH, o resultado no presente estudo torna-se preocupante, pois o pediatra e o obstetra, aparecem, com menor frequência em relaçãoao restante da equipe de saúde do HU que se destacou como fonte de informação. Os médicos devem fortalecer o vínculo com a gestante, uma vez que a consulta pré-natal é momento ideal para realização de orientações sobre aleitamento materno e doação de leite. Na literatura, no que se refere a fonte de informação sobre o serviço prestado pelo BLH, em primeiro lugar aparecem os serviços de saúde e a mídia aparece em segundo lugar como fonte de informação sobre o BLH (Pereira et. al., 2020). É urgente a conscientização e sensibilização desses profissionais de saúde quanto a necessidade de divulgação e apoio à doação de leite humano. De acordo com os autores, mulheres devidamente orientadas em relação a amamentação tem maior facilidade de identificar suas condições enquanto prováveis doadoras de leite materno ecompreendem melhor a relevância da sua doação para saúde pública (Machado, 2017).

\section{Considerações Finais}

Os resultados deste estudo permitiram identificar o perfil sociodemográfico e perinatal das doadoras de leite materno de um banco de leite humano no sul do Brasil, onde observou-se uma população de 200 nutrizes durante o estudo durante o ano de 2018. As características mais prevalentes foram em relação a idade das doadoras, que tinham em sua maioria mais de 25 anos, exerciam alguma atividade profissional em sua maioria. A maior parte das nutrizes realizou as consultas de pré-natal em instituições públicas, eram multíparas, com bebês nascidos a termo, de cesariana. Não possuíam experiência em gestação anterior com amamentação e receberam orientações sobre amamentação no pré-natal. No que se refere, as informações sobre o BLH, a maior parte das doadoras, recebeu essas informações de profissionais de saúde do hospital universitário.

Consideram-se como limitações do estudo a análise retrospectiva em apenas um ano, visto que, com um delineamento longitudinal retrospectivo, de dois ou mais anos, poderia-se analisar o aumento/diminuição do número de doadoras, as possíveis modificações de suas características e nas fontes de informação sobre o BLH. Ressaltamos também a limitação de produção científica sobre esse tema, impossibilitando contrastar alguns resultados do estudo de forma mais ampla, com a literatura.

Os resultados sugerem a necessidade de aprimorar e até mesmo ampliar os programas de saúde relacionados ao aleitamento materno, esclarecendo dúvidas pertinentes da população e elucidar o cenário brasileiro dos bancos de leite espalhados pelo país. Os achados desse estudo colaboram com o avanço científico para o ensino, a formação de novos profissionais de saúde, assim como a ampliação de novas pesquisas sobre a temática.

\section{Referências}

Anvisa. Agência Nacional de Vigilância Sanitária (2016). Banco de Leite Humano: Funcionamento, Prevenção e Controle de Riscos. Brasília. http://www20.anvisa.gov.br

Anvisa. Agência Nacional de Vigilância Sanitária (2020). Regulamento Técnico para o funcionamento de Bancos de Leite Humano. Brasília. http://www.bvsms.saude.gov.br

Barros, F. C. et al. (2018) Caesarean sections and the prevalence of preterm and early-term birtths Brazil:secondary analyses of national birth registration. BMJ Open.5 ago.

Brasil. Ministério da Saúde (2015). Saúde da Criança - Aleitamento Materno e Alimentação Complementar. Brasília. 2ed. Cadernos de Atenção Básica. n.23. http://bvsms.saude.gov.br/bvs/publicacoes/saude crianca aleitamento cab23.pdf

Brasil. Ministério da Saúde (2017) Diretrizes Nacionais de Assistência ao Parto Normal. Brasília. 1ed. http://bvsms.saude.gov.br/bvs/publicacoes/diretrizes_nacionais_assistencia_parto_normal.pdf

Daniels, B., Schmidt, S., King, T., Israel-Ballard, K., Amundson Mansen, K., \& Coutsoudis, A. (2017). The Effect of Simulated Flash-Heat Pasteurization on Immune Components of Human Milk Nutrients, 9(2), 178. https://doi.org/10.3390/nu9020178. 
Research, Society and Development, v. 11, n. 1, e46211125180, 2022

(CC BY 4.0) | ISSN 2525-3409 | DOI: http://dx.doi.org/10.33448/rsd-v11i1.25180

Dawod, B., \& Marshall, J. S. (2019). Cytokines and Soluble Receptors in Breast Milk as Enhancers of Ord Tolerance Development. Frontiers in Immunology, 10,16. https://doi.org/10.3389/fimmu.2019.00016

Fang, M. T., Grummer-Strawn, L., Maryuningsih, Y., \& Biller-Andomo, N. (2021). Human milk banks: A need for further evidence and guidance. The Lancet Global Health, 9(2), e104-e105. https://doi.org/10.1016/S2214-109X(20)30468-X.

Leal, M. C. et al. (2018) Saúde reprodutiva, materna, neonatal e infantil nos 30 anos do Sistema Único de Saúde (SUS). Ciência \& Saúde Coletiva; Rio de Janeiro v. 23, Ed. 6, p.1915-1928. http://www.search.proquest.com/openview

Lourenço, D., Bardini, G., Cunha, L. (2012) Perfil das doadoras do banco de leite humano do Hospital Nossa Senhora da Conceição, Tubarão/SC. Arquivos Catarin. de Medicina, 41(1)22-27. http://www.acm.org.br/revista/pdf/artigos/907.pdf

Machado, A. C. L., Santos, J. D. A., Trigueiros, P. Q. S. (2017) Perfil das doadoras de leite humano de uma maternidade federal da cidade de Salvador, Bahia. Rev. Ped. SOPERJ, v.17, n.2, p.18-24, jun. http://www.revistadepediatriasoperj.org.br

Maia, P. R. da S. et al. (2006). Rede Nacional de Bancos de Leite Humano: gênese e evolução. Revista Brasileira de Saúde Materno Infantil. Recife, v. 6, n. 3 , p. 285-292, jul./set. http://www.scielo.br/pdf/rbsmi/v6n3/1899.pdf

Mattar, M. J. G et al. (2011) Doação de leite humano: dificuldades e fatores limitantes. O Mundo da Saúde, 35(2):156-61.

Neia, V. J. C., Tavares, C. B. G., Ponhozi, I. B., Tiyo, B. T., Manin, L. P., Silveira, R. da, Chiavelli, L. U. R., Fuyama, F. A., Visentainer, L., Santos, O. O., Visentainer, J. E. L., \& Visentainer, J. V. (2021). Recommendations for donating breastmilk to human milk banks considering the Covid-19 pandemic. Research, Society and Development, 10 (8), e30210817258. http://doi.org/10.33448/rsd.V101817258.

Nunes, L.M. (2015) Importância do aleitamento materno na atualidade. Bol Cient Pediatr;04(3):55-8

Pereira, M. D., Aires, I. O., Emérito, L. L., Viana, V. M. O., França, C. C. S., Fernandes, C. F., Viana, V. A. O, \& Leal, D. L. (2020). Fatores associados à prevalência do aleitamento materno exclusivo ao neonato de baixo peso: revisão integrativa. Research, Society and Development, 9(10), e979108199. https://doi.org/10.33448/rsd-v9i10.8199.

Salvatori, G., De Rose, D.U., Amadio, P., Reposi, M.P., Piccioni, L., Concato, C., \& Dotta, A. (2021). Universal Screening for SARS-CoV-2 of all Human Milk Bank Samples. Journal of Human Lactation, 37(1), 40-42. https://doi.org/10.1177/0890334420962074.

Silva, D. I. S., Barbosa, A. L. O., Santana, A. L, Santos, R. V. C., Souza, V. C.G. B., Farias, J. V. C. e Farias, I. C. C. (2020). The importance of breastfeeding in the immunity of the newborn. Research, Society and Development, 9 (7):1-14, e664974629. 